\title{
COMPARISON OF TWO NOTIONS OF WEAK CROSSED PRODUCT
}

\author{
JORGE A. GUCCIONE AND JUAN J. GUCCIONE
}

\begin{abstract}
We compare the restriction to the context of weak Hopf algebras of the notion of crossed product with a Hopf algebroid introduced in 6$]$ with the notion of crossed product with a weak Hopf algebra introduced in [5].
\end{abstract}

\section{Contents}

1 Preliminaries . . . . . . . . . . . . . . . . . . . . . . . . . . 1

1.1 Weak Hopf algebras . . . . . . . . . . . . . . . . . . . . . 2

1.2 Crossed product $A \#_{\sigma}^{\rho} H$. . . . . . . . . . . . . . . . . . . . . . . . . . 2

1.3 Crossed product $A \times \times_{\varsigma}^{\rho} H$. . . . . . . . . . . . . . . . . . . . . . . . . . . . . 4

2 The comparison . . . . . . . . . . . . . . . . . . . . . . . . . 5

2.1 Crossed products $A \#_{\sigma}^{\rho} H$ that induce crossed products $A \times_{\varsigma}^{\rho} H$. . . . . . 5

2.2 Crossed product $A \#_{\sigma}^{\rho} H$ associated with a crossed product $A \times{ }_{\varsigma}^{\rho} H$. . . . 6

2.3 The isomorphism . . . . . . . . . . . . . . . . . . . . . . 8

\section{Introduction}

Two different notions of crossed product of an algebra $A$ with a weak Hopf algebra $H$ have been purposed. The first one is the restriction to the context of weak Hopf algebras of the notion of crossed product with a Hopf algebroid introduced by Böhm and Brzeziński in [6. The second one was introduced in 5] and studied in a series of papers (see for instance [2 4, 9, 12]). The aim of this paper is to prove that there exists the last one if and only if there exists the first one and condition (10) below is fulfilled. Moreover, in this case both constructions are canonically isomorphic. Finally we provide an example which shows that there are crossed products of algebras with weak Hopf algebras in the sense of Böhm and Brzeziński that not satisfy condition (10).

\section{Preliminaries}

In this section we review the notion of weak Hopf algebra and the notions of crossed product that we want to compare.

2010 Mathematics Subject Classification. primary 16T05; secondary 18D10.

Key words and phrases. Weak Hopf algebras, Weak crossed products.

Jorge A. Guccione and Juan J. Guccione were supported by UBACyT 20020150100153BA (UBA) 


\subsection{Weak Hopf algebras}

Weak bialgebras and weak Hopf algebras are generalizations of bialgebras and Hopf algebras, introduced in 7, 8, in which the axioms about the unit, the counit and the antipode are replaced by weaker properties. Next we give a brief review of the basic properties of these structures.

Let $k$ be a field. A weak bialgebra is a $k$-vector space $H$, endowed with an algebra structure and a coalgebra structure, such that $\Delta(h l)=\Delta(h) \Delta(l)$ for all $h, l \in H$, and the equalities

$$
\Delta^{2}(1)=1^{(1)} \otimes 1^{(2)} 1^{\left(1^{\prime}\right)} \otimes 1^{\left(2^{\prime}\right)}=1^{(1)} \otimes 1^{\left(1^{\prime}\right)} 1^{(2)} \otimes 1^{\left(2^{\prime}\right)}
$$

and

$$
\epsilon(h l m)=\epsilon\left(h l^{(1)}\right) \epsilon\left(l^{(2)} m\right)=\epsilon\left(h l^{(2)}\right) \epsilon\left(l^{(1)} m\right) \quad \text { for all } h, l, m \in H,
$$

are fulfilled, where we are using the Sweedler notation for the coproduct, with the summation symbol omitted. A weak bialgebra morphism is a function $g: H \rightarrow L$ that is an algebra and a coalgebra map. For each weak bialgebra $H$, the maps $\Pi^{L}: H \rightarrow H$ and $\Pi^{R}: H \rightarrow H$ defined by

$$
\Pi^{L}(h):=\epsilon\left(1^{(1)} h\right) 1^{(2)} \text { and } \Pi^{R}(h):=1^{(1)} \epsilon\left(h 1^{(2)}\right),
$$

respectively, are idempotent. We set $H^{L}:=\operatorname{Im}\left(\Pi^{L}\right)$ and $H^{R}:=\operatorname{Im}\left(\Pi^{R}\right)$. In 77 it was proven that $H^{L}$ and $H^{R}$ are subalgebras of $H$.

Let $H$ be a weak bialgebra. An antipode of $H$ is a map $S: H \rightarrow H$ (or $S_{H}$ if necessary to avoid confusion), such that $h^{(1)} S\left(h^{(2)}\right)=\Pi^{L}(h), S\left(h^{(1)}\right) h^{(2)}=\Pi^{R}(h)$ and $S\left(h^{(1)}\right) h^{(2)} S\left(h^{(3)}\right)=S(h)$, for all $h \in H$. As it was shown in [7, an antipode $S$, if there exists, is unique. It was also shown in 7 that $S$ is antimultiplicative, anticomultiplicative and leaves the unit and counit invariant. A weak Hopf algebra is a weak bialgebra that has an antipode. A morphism of weak Hopf algebras $g: H \rightarrow L$ is simply a bialgebra morphism from $H$ to $L$. In [1, Proposition 1.4] it was proven that if $g: H \rightarrow L$ is a weak Hopf algebra morphism, then $g \circ S_{H}=S_{L} \circ g$. From now one we assume that $H$ is a weak Hopf algebra with bijective antipode.

\subsection{Crossed product $A \#_{\sigma}^{\rho} H$}

The notion of crossed product with a Hopf algebroid was introduced by Böhm and Brzeziński in 6. Since weak Hopf algebras provide examples of Hopf algebroids, this gives a notion of crossed product with a weak Hopf algebra. In this subsection we review this construction. For the proofs of the results we refer to 6 (see also [1] ).

Let $A$ be a $k$-algebra, $H$ a weak Hopf algebra and $\rho: H \otimes A \rightarrow A$ a map. We set $h \cdot a:=\rho(h \otimes a)$.

Definition 1.1. We say that $H$ measures $A$ via $\rho$ and that $\rho$ is a measuring if

(1) $h \cdot 1_{A}=\Pi^{L}(h) \cdot 1_{A}$,

(2) $h \cdot\left(a a^{\prime}\right)=\left(h^{(1)} \cdot a\right)\left(h^{(2)} \cdot a^{\prime}\right)$,

(3) $S^{-1}(l) h \cdot a=(h \cdot a)\left(l \cdot 1_{A}\right)$ and $l h \cdot a=\left(l \cdot 1_{A}\right)(h \cdot a)$

for all $h \in H, l \in H^{L}$ and $a, a^{\prime} \in A$. The measuring is unital if

(4) $1 \cdot a=a$ for all $a \in A$.

By items (3) and (4) we have

$$
h \cdot(k \cdot a)=h k \cdot a \quad \text { for all } h \in H^{L} H^{R}, k \in H \text { and } a \in A .
$$

In the rest of this subsection

- we assume that $\rho$ is a unital measuring,

- given a map $\sigma: H \otimes_{H^{R}} H \rightarrow A$ we write $\sigma(h, k)$ instead of $\sigma\left(h \otimes_{H^{R}} k\right)$, 
- given a map $\bar{\sigma}: H \otimes_{H^{L}} H \rightarrow A$ we write $\bar{\sigma}(h, k)$ instead of $\bar{\sigma}\left(h \otimes_{H^{L}} k\right)$.

Definition 1.2. A map $\sigma: H \otimes_{H^{R}} H \rightarrow A$ is a normal cocycle if

(5) $\sigma(l h, k)=\left(l \cdot 1_{A}\right) \sigma(h, k)$ and $\sigma\left(S^{-1}(l) h, k\right)=\sigma(h, k)\left(l \cdot 1_{A}\right)$,

(6) $\left(h^{(1)} \cdot\left(l \cdot 1_{A}\right)\right) \sigma\left(h^{(2)}, k\right)=\sigma(h, l k)$,

(7) $\sigma(1, h)=\sigma(h, 1)=h \cdot 1_{A}$,

(8) $\left(h^{(1)} \cdot \sigma\left(k^{(1)}, m^{(1)}\right)\right) \sigma\left(h^{(2)}, k^{(2)} m^{(2)}\right)=\sigma\left(h^{(1)}, k^{(1)}\right) \sigma\left(h^{(2)} k^{(2)}, m\right)$,

for all $h, k, m \in H$ and $l \in H^{L}$.

Definition 1.3. A map $\sigma: H \otimes_{H^{R}} H \rightarrow A$ satisfy the twisted module condition if

(9) $\left(h^{(1)} \cdot\left(k^{(1)} \cdot a\right)\right) \sigma\left(h^{(2)}, k^{(2)}\right)=\sigma\left(h^{(1)}, k^{(1)}\right)\left(h^{(2)} k^{(2)} \cdot a\right)$ for all $h, k \in H$ and $a \in A$.

Proposition 1.4. The following facts hold:

- $h \cdot(k \cdot a)=h k \cdot a$ for all $h \in H^{L} H^{R}, k \in H$ and $a \in A$.

- $\sigma(h, k)=\sigma\left(h^{(1)}, k^{(1)}\right)\left(h^{(2)} k^{(2)} \cdot 1_{A}\right)$ for all $h, k \in H$.

Proof. The first item follows easily from items (3) and (4). We next prove the second one. By items (1), (6) and (9), we have

$$
\begin{aligned}
\sigma\left(h^{(1)}, k^{(1)}\right)\left(h^{(2)} k^{(2)} \cdot 1_{A}\right) & =\left(h^{(1)} \cdot\left(k^{(1)} \cdot 1_{A}\right) \sigma\left(h^{(2)}, k^{(2)}\right)\right. \\
& =\left(h^{(1)} \cdot\left(\Pi^{L}\left(k^{(1)}\right) \cdot 1_{A}\right) \sigma\left(h^{(2)}, k^{(2)}\right)\right. \\
& =\sigma\left(h, \Pi^{L}\left(k^{(1)}\right) k^{(2)}\right) .
\end{aligned}
$$

Since $\Pi^{L}\left(k^{(1)}\right) k^{(2)}=k$ this ends the proof.

Theorem 1.5. Let $\sigma: H \otimes_{H^{R}} H \rightarrow A$ be a map satisfying conditions (5) and (6) of Definition 1.2. Consider $A$ a right $H^{L}$-module via $a \cdot l:=a\left(l \cdot 1_{A}\right)$. The $k$-vector space $A \otimes_{H^{L}} H$ is an associative algebra with unit $1_{A} \otimes_{H^{L}} 1$ via

$$
\left(a \otimes_{H^{L}} h\right)\left(b \otimes_{H^{L}} k\right):=a\left(h^{(1)} \cdot b\right) \sigma\left(h^{(2)}, k^{(1)}\right) \otimes_{H^{L}} h^{(3)} k^{(2)}
$$

if and only if the measuring $\rho$ is unital and $\sigma$ is a normal cocycle that satisfies the twisted module condition.

Definition 1.6. The algebra constructed in Theorem 1.5 is denoted $A \#_{\sigma}^{\rho} H$ and is called the weak crossed product of $A$ with $H$ associated with $\rho$ and $\sigma$.

We consider $A \#_{\sigma}^{\rho} H$ as a left $A$-module via the natural action and as a right $H$-comodule via the map $\delta: A \#_{\sigma}^{\rho} H \rightarrow A \#_{\sigma}^{\rho} H \otimes H$, defined by $\delta\left(a \otimes_{H^{L}} h\right):=a \otimes_{H^{L}} h^{(1)} \otimes h^{(2)}$.

Proposition 1.7. Under conditions (1), (2), (6) and (7) the following assertions are equivalent

(10) $h \cdot\left(l \cdot 1_{A}\right)=h l \cdot 1_{A}$ for all $h \in H$ and $l \in H^{L}$,

(11) $h \cdot\left(l \cdot 1_{A}\right)=h l \cdot 1_{A}$ for all $h, l \in H$,

(12) $\sigma(h, l)=\sigma(h l, 1)$ for all $h \in H$ and $l \in H^{L}$.

Proof. (1) $\Leftrightarrow(2)$. Since, by item (1) and [7, Equality (2.5a)], we have

$$
h l \cdot 1_{A}=\Pi^{L}(h l) \cdot 1_{A}=\Pi^{L}\left(h \Pi^{L}(l)\right) \cdot 1_{A}=h \Pi^{L}(l) \cdot 1_{A} \quad \text { and } \quad h \cdot\left(\Pi^{L}(l) \cdot 1_{A}\right)=h \cdot\left(l \cdot 1_{A}\right),
$$

for all $h, l \in H$.

$(1) \Leftrightarrow(3)$ Since, by items (2), (6) and (7), we have $\sigma(h, l)=\left(h^{(1)} \cdot\left(l \cdot 1_{A}\right)\right) \sigma\left(h^{(2)}, 1\right)=\left(h^{(1)} \cdot\left(l \cdot 1_{A}\right)\right)\left(h^{(2)} \cdot 1_{A}\right)=h \cdot\left(l \cdot 1_{A}\right) \quad$ and $\quad h l \cdot 1_{A}=\sigma(h l, 1)$. for all $h$ and $l \in H^{L}$. 
Definition 1.8. We say that a normal cocycle $\sigma: H \otimes_{H^{R}} H \rightarrow A$ satisfying the twisted module condition is invertible if there exists a map $\bar{\sigma}: H \otimes_{H^{L}} H \rightarrow A$ satisfying

(13) $\bar{\sigma}(h, k)=\left(h^{(1)} k^{(1)} \cdot 1_{A}\right) \bar{\sigma}\left(h^{(2)}, k^{(2)}\right)$,

(14) $\bar{\sigma}(l h, k)=\left(l \cdot 1_{A}\right) \bar{\sigma}(h, k)$ and $\bar{\sigma}\left(S^{-1}(l) h, k\right)=\bar{\sigma}(h, k)\left(l \cdot 1_{A}\right)$,

(15) $\bar{\sigma}\left(h^{(1)}, k\right)\left(h^{(2)} \cdot\left(l \cdot 1_{A}\right)\right)=\bar{\sigma}\left(h, S^{-1}(l) k\right)$,

(16) $\sigma\left(h^{(1)}, k^{(1)}\right) \bar{\sigma}\left(h^{(2)}, k^{(2)}\right)=h \cdot\left(k \cdot 1_{A}\right)$ and $\bar{\sigma}\left(h^{(1)}, k^{(1)}\right) \sigma\left(h^{(2)}, k^{(2)}\right)=h k \cdot 1_{A}$,

for all $h, k \in H$ and $l \in H^{L}$.

Remark 1.9. By item (13) and (16), we have

$$
\begin{aligned}
\bar{\sigma}\left(h^{(1)}, k^{(1)}\right)\left(h^{(2)} \cdot\left(k^{(2)} \cdot 1_{A}\right)\right) & =\bar{\sigma}\left(h^{(1)}, k^{(1)}\right) \sigma\left(h^{(2)}, k^{(2)}\right) \bar{\sigma}\left(h^{(3)}, k^{(3)}\right) \\
& =\left(h^{(1)} k^{(1)} \cdot 1_{A}\right) \bar{\sigma}\left(h^{(2)}, k^{(2)}\right) \\
& =\bar{\sigma}(h, k) .
\end{aligned}
$$

An standard computation using this shows that $\bar{\sigma}$, which is called the inverse of $\sigma$, is unique.

Remark 1.10. It $\bar{\sigma}: H \otimes_{H^{L}} H \rightarrow A$ satisfies items (14)-(16), then the map $\tilde{\sigma}: H \otimes_{H^{L}} H \rightarrow A$, defined by $\tilde{\sigma}(h, k):=\left(h^{(1)} k^{(1)} \cdot 1_{A}\right) \bar{\sigma}\left(h^{(2)}, k^{(2)}\right)$, satisfies items (13)-(16).

$$
\begin{aligned}
\tilde{\sigma}(h, k) & =\left(h^{(1)} k^{(1)} \cdot 1_{A}\right) \bar{\sigma}\left(h^{(2)}, k^{(2)}\right) \\
& =\left(h^{(1)} k^{(1)} \cdot 1_{A}\right)\left(h^{(2)} k^{(2)} \cdot 1_{A}\right) \bar{\sigma}\left(h^{(3)}, k^{(3)}\right) \\
& =\left(h^{(1)} k^{(1)} \cdot 1_{A}\right) \tilde{\sigma}\left(h^{(2)}, k^{(2)}\right) .
\end{aligned}
$$

\subsection{Crossed product $A \times \times_{\varsigma}^{\rho} H$}

In this subsection we review the construction of weak crossed products over weak Hopf algebras introduced in [5]. As in the previous subsection $A$ is a $k$-algebra, $H$ is a weak Hopf algebra, $\rho: H \otimes A \rightarrow A$ is a map and we set $h \cdot a:=\rho(h \otimes a)$.

Definition 1.11. We say that $A$ is a left weak $H$-module algebra via $\rho$, if it satisfies conditions (2), (4) and (11). In this case we say that $\rho$ is a left weak action of $H$ on $A$.

In the rest of this subsection we assume that $A$ is a left weak $H$-module algebra via $\rho$.

Definition 1.12. Let $\varsigma: H \otimes H \longrightarrow A$ be a map. We say that:

(17) $\varsigma$ is a cocycle if

$$
\left(h^{(1)} \cdot \varsigma\left(k^{(1)} \otimes m^{(1)}\right)\right) \varsigma\left(h^{(2)} \otimes k^{(2)} m^{(2)}\right)=\varsigma\left(h^{(1)} \otimes k^{(1)}\right) \varsigma\left(h^{(2)} k^{(2)} \otimes m\right) \quad \text { for all } h, k, m \in H .
$$

(18) $\varsigma$ satisfies the twisted module condition if

$$
\left(h^{(1)} \cdot\left(k^{(1)} \cdot a\right)\right) \varsigma\left(h^{(2)} \otimes k^{(2)}\right)=\varsigma\left(h^{(1)} \otimes k^{(1)}\right)\left(h^{(2)} k^{(2)} \cdot a\right) \quad \text { for all } h, k \in H \text { and } a \in A .
$$

Let $\nu: k \rightarrow A \otimes H$ be the map defined by $\nu(\lambda):=\lambda 1^{(1)} \cdot 1_{A} \otimes 1^{(2)}$. In the sequel we assume that

(19) $\varsigma(h \otimes k)=\varsigma\left(h^{(1)} \otimes k^{(1)}\right)\left(h^{(2)} k^{(2)} \cdot 1_{A}\right)$,

(20) $h \cdot 1_{A}=\left(h^{(1)} \cdot\left(1^{(1)} \cdot 1_{A}\right)\right) \varsigma\left(h^{(2)} \otimes 1^{(2)}\right)$,

(21) $h \cdot 1_{A}=\left(1^{(1)} \cdot 1_{A}\right) \varsigma\left(1^{(2)} \otimes h\right)$,

(22) $a\left(1^{(1)} \cdot 1_{A}\right) \otimes 1^{(2)}=1^{(1)} \cdot a \otimes 1^{(2)}$, 
for all $h, k \in H$ and $a \in A$.

Remark 1.13. Under conditions (18) and (19), conditions (20) and (21) are equivalent to the fact that $\varsigma(1 \otimes h)=\varsigma(h \otimes 1)=h \cdot 1_{A}$ for all $h \in H^{1}$ (see 10, Remarks 2.17 and 2.19]).

Remark 1.14. From condition (11), (18) and (19) it follows that

$\left(h^{(1)} k^{(1)} \cdot 1_{A}\right) \varsigma\left(h^{(2)} \otimes k^{(2)}\right)=\left(h^{(1)} \cdot\left(k^{(1)} \cdot 1_{A}\right)\right) \varsigma\left(h^{(2)} \otimes k^{(2)}\right)=\varsigma\left(h^{(1)} \otimes k^{(1)}\right)\left(h^{(2)} k^{(2)} \cdot 1_{A}\right)=\varsigma(h \otimes k)$.

for all $h, k \in H$

Let $A \times H$ be the image of the map $\nabla_{\rho}: A \otimes H \rightarrow A \otimes H$, defined by $\nabla_{\rho}(a \otimes h):=a\left(h^{(1)} \cdot 1_{A}\right) \otimes h^{(2)}$. From now on, for each simple tensor $a \otimes h \in A \otimes H$, we set $a \times h:=\nabla_{\rho}(a \otimes h)$.

Theorem 1.15. Under conditions (2), (4), (11) and (17)-(25), the $k$-vector space $A \times H$ is an associative algebra with unit $1_{A} \times 1$, via

$$
(a \times h)(b \times k):=a\left(h^{(1)} \cdot b\right) \varsigma\left(h^{(2)} \otimes k^{(1)}\right) \times h^{(3)} k^{(2)} .
$$

Definition 1.16. The algebra constructed in Theorem 1.15 is denoted $A \times{ }_{\varsigma}^{\rho} H$ and is called the weak crossed product of $A$ with $H$ associated with $\rho$ and $\varsigma$.

We consider $A \times{ }_{\varsigma}^{\rho} H$ as a left $A$-module via the natural action and as a right $H$-comodule via the map $\delta: A \times_{\varsigma}^{\rho} H \rightarrow A \times \times_{\varsigma}^{\rho} H \otimes H$, defined by $\delta(a \times h):=\left(a \times h^{(1)}\right) \otimes h^{(2)}$.

Definition 1.17. Assume that conditions (2), (4), (11) and (17)-(25) are fulfilled. We say that $\varsigma: H \otimes H \rightarrow A$ is invertible if there exists a map $\bar{\varsigma}: H \otimes H \rightarrow A$ satisfying

$(23) \bar{\varsigma}(h, k)=\left(h^{(1)} k^{(1)} \cdot 1_{A}\right) \bar{\varsigma}\left(h^{(2)} \otimes k^{(2)}\right)$,

$(24) \varsigma\left(h^{(1)} \otimes k^{(1)}\right) \bar{\varsigma}\left(h^{(2)} \otimes k^{(2)}\right)=\bar{\varsigma}\left(h^{(1)} \otimes k^{(1)}\right) \varsigma\left(h^{(2)} \otimes k^{(2)}\right)=h k \cdot 1_{A}$,

for all $h, k \in H$.

The map $\bar{\varsigma}$ is unique, satisfies $\bar{\varsigma}(h \otimes k)=\left(h^{(1)} k^{(1)} \cdot 1_{A}\right) \bar{\varsigma}\left(h^{(2)} \otimes k^{(2)}\right)$ and is called the inverse of $\varsigma$ (see the discussion below 10, Definition 5.6]).

\section{The comparison}

Let $A$ be a $k$-algebra, $H$ be a weak Hopf algebra and $\rho: H \otimes A \rightarrow A$ a map. As in Subsections 1.2 and 1.3 we set $h \cdot a:=\rho(h \otimes a)$.

\subsection{Crossed products $A \#_{\sigma}^{\rho} H$ that induce crossed products $A \times{ }_{\varsigma}^{\rho} H$}

The aim of this subsection is to prove the following result:

Theorem 2.1. Let $\sigma: H \otimes_{H^{R}} H \rightarrow A$ be a map and let $\varsigma:=\sigma \circ p$, where $p: H \otimes H \rightarrow H \otimes_{H^{R}} H$ is the canonical surjection. If the maps $\rho$ and $\sigma$ satisfy conditions (1)-(10) then $\rho$ and $\varsigma$ satisfy conditions (2), (4), (11) and (17)-(22).

From here to the end of this subsection we assume that conditions (1)-(10) are fulfilled.

Proof of Theorem 2.1. Items (2) and (4) are trivially fulfilled. By Proposition 1.7, item (11) is fulfilled. Items (17) and (18) follow from items (8) and (9) respectively, while item (19) follows from Proposition 1.4. By Proposition 1.7 and items (2), (7) and (10), we have

$$
\left(h^{(1)} \cdot\left(1^{(1)} \cdot 1_{A}\right)\right) \varsigma\left(h^{(2)} \otimes 1^{(2)}\right)=\left(h^{(1)} 1^{(1)} \cdot 1_{A}\right) \sigma\left(h^{(2)} 1^{(2)}, 1\right)=\left(h^{(1)} \cdot 1_{A}\right)\left(h^{(2)} \cdot 1_{A}\right)=h \cdot 1_{A},
$$

\footnotetext{
${ }^{1} \mathrm{~A}$ map $\varsigma$ satisfying this condition is called normal.
} 
which proves that item (20) holds. By items (4), (6) and (7), we have

$$
\left(1^{(1)} \cdot 1_{A}\right) \varsigma\left(1^{(2)} \otimes h\right)=\left(1^{(1)} \cdot\left(1 \cdot 1_{A}\right)\right) \sigma\left(1^{(2)}, h\right)=\sigma(1, h)=h \cdot 1_{A},
$$

which proves item (21). Finally, in order to check item (22), it suffices to note that by Equality 1.1) and items (2) and (11),

$$
\begin{aligned}
\left(1^{(1)} \cdot 1_{A}\right)\left(1^{(2)} \cdot a\right) \otimes 1^{(3)} & =\left(1^{(1)} \cdot a\right)\left(1^{(2)} \cdot 1_{A}\right) \otimes 1^{(3)} \\
& =\left(1^{(1)} \cdot a\right)\left(1^{(2)} \cdot\left(1^{\left(1^{\prime}\right)} \cdot 1_{A}\right)\right) \otimes 1^{\left(2^{\prime}\right)} \\
& =a\left(1^{(1)} \cdot 1_{A}\right) \otimes 1^{(2)}
\end{aligned}
$$

as desired.

Proposition 2.2. Under the hypotheses of Theorem 2.1. if $\sigma$ is invertible, then $\varsigma$ is also.

Proof. Let $\bar{\sigma}$ be the inverse of $\sigma$ and let $\tilde{\varsigma}:=\bar{\sigma} \circ q$, where $q: H \otimes H \rightarrow H \otimes_{H^{L}} H$ is the canonical surjection. From items (11), (13) and (16) it follows that $\tilde{\varsigma}$ satisfies items (23) and (24).

\subsection{Crossed product $A \#_{\sigma}^{\rho} H$ associated with a crossed product $A \times{ }_{\varsigma}^{\rho} H$}

The aim of this subsection is to prove the following result:

Theorem 2.3. Let $\varsigma: H \otimes H \rightarrow A$ be a map. If the maps $\rho$ and $\varsigma$ satisfy conditions (2), (4), (11) and (17)-(22), then $\varsigma$ factorizes throughout a map $\sigma: H \otimes_{H^{R}} H \rightarrow A$ and the maps $\rho$ and $\sigma$ satisfy conditions (1)-(9).

From here to the end of this subsection we assume that conditions (2), (4), (11) and (17)-(22) are fulfilled.

Lemma 2.4. The equality $k \cdot(h \cdot a)=k h \cdot a$ holds for all $k \in H^{L} \cup H^{R}, h \in H$ and $a \in A$.

Proof. We have,

$$
\begin{aligned}
k \cdot(h \cdot a) & =k^{(1)} \cdot\left(h^{(1)} \cdot a\right)\left(k^{(2)} h^{(2)} \cdot 1_{A}\right) \\
& =k^{(1)} \cdot\left(h^{(1)} \cdot a\right) \varsigma\left(k^{(2)} \otimes h^{(2)}\right) \\
& =\varsigma\left(k^{(1)} \otimes h^{(1)}\right)\left(k^{(2)} h^{(2)} \cdot a\right) \\
& =\left(k^{(1)} h^{(1)} \cdot 1_{A}\right)\left(k^{(2)} h^{(2)} \cdot a\right) \\
& =k h \cdot a .
\end{aligned}
$$

where the first equality follows from items (2) and (11); the second and the fourth ones, from Remark 1.13, the fact that $\Delta(k) \in H^{R} H^{L} \otimes H^{R} H^{L}$ and 10 . Propositions 2.7 and 2.8]; the third one, from item (18); and the last one, from item (2).

Lemma 2.5. For all $h, k \in H$,

$$
\left(h^{(1)} \cdot 1_{A}\right) \varsigma\left(h^{(2)} \otimes k\right)=\varsigma\left(h^{(1)} \otimes k\right)\left(h^{(2)} \cdot 1_{A}\right)=\varsigma(h \otimes k) .
$$

Proof. By Remark 1.14 and item (2),

$$
\begin{aligned}
\left(h^{(1)} \cdot 1_{A}\right) \varsigma\left(h^{(2)} \otimes k\right) & =\left(h^{(1)} \cdot 1_{A}\right)\left(h^{(2)} \cdot\left(k^{(1)} \cdot 1_{A}\right) \varsigma\left(h^{(3)} \otimes k^{(2)}\right)\right. \\
& =\left(h^{(1)} \cdot\left(k^{(1)} \cdot 1_{A}\right) \varsigma\left(h^{(2)} \otimes k^{(2)}\right)\right. \\
& =\varsigma(h \otimes k),
\end{aligned}
$$


and by items (2), (11) and (19),

$$
\begin{aligned}
\varsigma\left(h^{(1)} \otimes k\right)\left(h^{(2)} \cdot 1_{A}\right) & =\varsigma\left(h^{(1)} \otimes k^{(1)}\right)\left(h^{(2)} k^{(2)} \cdot 1_{A}\right)\left(h^{(3)} \cdot 1_{A}\right) \\
& =\varsigma\left(h^{(1)} \otimes k^{(1)}\right)\left(h^{(2)} \cdot\left(k^{(2)} \cdot 1_{A}\right)\right)\left(h^{(3)} \cdot 1_{A}\right) \\
& =\varsigma\left(h^{(1)} \otimes k^{(1)}\right)\left(h^{(2)} \cdot\left(k^{(2)} \cdot 1_{A}\right)\right) \\
& =\varsigma\left(h^{(1)} \otimes k^{(1)}\right)\left(h^{(2)} k^{(2)} \cdot 1_{A}\right) \\
& =\varsigma(h \otimes k),
\end{aligned}
$$

which finishes the proof.

Remark 2.6. By Remark 1.14

$\epsilon\left(h^{(1)} l^{(1)}\right) \varsigma\left(h^{(2)} \otimes l^{(2)}\right)=\epsilon\left(h^{(1)} l^{(1)}\right)\left(h^{(2)} l^{(2)} \cdot 1_{A}\right) \varsigma\left(h^{(3)} \otimes l^{(3)}\right)=\left(h^{(1)} l^{(1)} \cdot 1_{A}\right) \varsigma\left(h^{(2)} \otimes l^{(2)}\right)=\varsigma(h \otimes l)$.

Proof of Theorem 2.3. By item (19) and 10, Propositions 2.4 and 2.7], the map $\sigma$ exists. Items (2) and (4) are trivially fulfilled. By items (17) and (18), items (8) and (9) hold. Moreover by 10 , Proposition 4.2] and items (2), (4) and (11), item (1) also hold. Let $\bar{\Pi}^{L}:=S^{-1} \circ \Pi^{L}$ and $l \in H^{L}, h \in H$ and $a \in A$. By items (2), (4) and (11), Lemma 2.4 and 10 , Propositions 4.1 and 4.2], we have

and

$$
\left(l \cdot 1_{A}\right)(h \cdot a)=l \cdot(h \cdot a)=l h \cdot a
$$

$$
(h \cdot a)\left(l \cdot 1_{A}\right)=\bar{\Pi}^{L}(l) \cdot(h \cdot a)=S^{-1}(l) \cdot(h \cdot a)=S^{-1}(l) h \cdot a,
$$

which proves item (3). In order to check item (5), it is sufficient to note that, by Lemma 2.5 . 7. Equalities (2.7a) and (2.7b)], and item (3),

and

$$
\begin{aligned}
\sigma(l h, k) & =\left(l^{(1)} h^{(1)} \cdot 1_{A}\right) \sigma\left(l^{(2)} h^{(2)}, k\right) \\
& =\left(l h^{(1)} \cdot 1_{A}\right) \sigma\left(h^{(2)}, k\right) \\
& =\left(l \cdot 1_{A}\right)\left(h^{(1)} \cdot 1_{A}\right) \sigma\left(h^{(2)}, k\right) \\
& =\left(l \cdot 1_{A}\right) \sigma(h, k),
\end{aligned}
$$

$$
\begin{aligned}
\sigma\left(S^{-1}(l) h, k\right) & =\sigma\left(S^{-1}(l)^{(1)} h^{(1)}, k\right)\left(S^{-1}(l)^{(2)} h^{(2)} \cdot 1_{A}\right) \\
& =\sigma\left(h^{(1)}, k\right)\left(S^{-1}(l) h^{(2)} \cdot 1_{A}\right) \\
& =\sigma\left(h^{(1)}, k\right)\left(h^{(2)} \cdot 1_{A}\right)\left(l \cdot 1_{A}\right) \\
& =\sigma(h, k)\left(l \cdot 1_{A}\right) .
\end{aligned}
$$

By item (11), 7, Equality (2.7a)], Lemma 2.5 and 10, Proposition 2.8] (which applies by Remark 2.6), we have

$$
\left(h^{(1)} \cdot\left(l \cdot 1_{A}\right)\right) \sigma\left(h^{(2)}, k\right)=\left(h^{(1)} l^{(1)} \cdot 1_{A}\right) \sigma\left(h^{(2)} l^{(2)}, k\right)=\sigma(h l, k)=\sigma(h, l k),
$$

for all $l \in H^{L}$ and $h, k \in H$. So, item (6) holds. Finally, item (7) follows from Remark 1.13 and items (20) and (21).

Proposition 2.7. Under the hypotheses of Theorem 2.3, if $\varsigma$ is invertible, then $\sigma$ is also.

Proof. Let $\bar{\varsigma}$ be the inverse of $\varsigma$. By 10 , Proposition 5.13], the map $\bar{\varsigma}$ factorizes throughout a map $\bar{\sigma}: H \otimes_{H^{L}} H \rightarrow A$. Item (13) follows immediately from item (23), while item (16) follows from items (11) and (24). We next prove item (14). By item (11) and Remark 1.9, we have

$$
\bar{\sigma}(h, k)=\bar{\sigma}\left(h^{(1)}, k^{(1)}\right)\left(h^{(2)} k^{(2)} \cdot 1_{A}\right) \quad \text { for all } h, k \in H .
$$


Using this, items (3), (13) and 77, Equalities (2.7a) and (2.7b)], we obtain that

$$
\begin{aligned}
\bar{\sigma}(l h, k) & =\left(l^{(1)} h^{(1)} k^{(1)} \cdot 1_{A}\right) \bar{\sigma}\left(l^{(2)} h^{(2)}, k^{(2)}\right) \\
& =\left(l h^{(1)} k^{(1)} \cdot 1_{A}\right) \bar{\sigma}\left(h^{(2)}, k^{(2)}\right) \\
& =\left(l \cdot 1_{A}\right)\left(h^{(1)} k^{(1)} \cdot 1_{A}\right) \bar{\sigma}\left(h^{(2)}, k^{(2)}\right) \\
& =\left(l \cdot 1_{A}\right) \bar{\sigma}(h, k)
\end{aligned}
$$

and

$$
\begin{aligned}
\bar{\sigma}\left(S^{-1}(l) h, k\right) & =\bar{\sigma}\left(S^{-1}(l)^{(1)} h^{(1)}, k^{(1)}\right)\left(S^{-1}(l)^{(2)} h^{(2)} k^{(2)} \cdot 1_{A}\right) \\
& =\bar{\sigma}\left(h^{(1)}, k^{(1)}\right)\left(S^{-1}(l) h^{(2)} k^{(2)} \cdot 1_{A}\right) \\
& =\bar{\sigma}\left(h^{(1)}, k^{(1)}\right)\left(h^{(2)} k^{(2)} \cdot 1_{A}\right)\left(l \cdot 1_{A}\right) \\
& =\bar{\sigma}(h, k)\left(l \cdot 1_{A}\right)
\end{aligned}
$$

for all $h, k \in H$ and $l \in H^{L}$, which is item (14). It remains to prove item (15). By the first equality in [2.3), 7, Equality (2.7b)] and items (2), (3) and (11), we obtain that

$$
\begin{aligned}
\bar{\sigma}\left(h, S^{-1}(l) k\right) & =\bar{\sigma}\left(h^{(1)}, S^{-1}(l)^{(1)} k^{(1)}\right)\left(h^{(2)} S^{-1}(l)^{(2)} k^{(2)} \cdot 1_{A}\right) \\
& =\bar{\sigma}\left(h^{(1)}, k^{(1)}\right)\left(h^{(2)} S^{-1}(l) k^{(2)} \cdot 1_{A}\right) \\
& =\bar{\sigma}\left(h^{(1)}, k^{(1)}\right)\left(h^{(2)} \cdot\left(S^{-1}(l) k^{(2)} \cdot 1_{A}\right)\right) \\
& =\bar{\sigma}\left(h^{(1)}, k^{(1)}\right)\left(h^{(2)} \cdot\left(\left(k^{(2)} \cdot 1_{A}\right)\left(l \cdot 1_{A}\right)\right)\right) \\
& =\bar{\sigma}\left(h^{(1)}, k^{(1)}\right)\left(h^{(2)} \cdot\left(k^{(2)} \cdot 1_{A}\right)\right)\left(h^{(3)} \cdot\left(l \cdot 1_{A}\right)\right) \\
& =\bar{\sigma}\left(h^{(1)}, k^{(1)}\right)\left(h^{(2)} k^{(2)} \cdot 1_{A}\right)\left(h^{(3)} \cdot\left(l \cdot 1_{A}\right)\right) \\
& =\bar{\sigma}\left(h^{(1)}, k\right)\left(h^{(2)} \cdot\left(l \cdot 1_{A}\right)\right)
\end{aligned}
$$

for all $h, k \in H$ and $l \in H^{L}$, as desired.

\subsection{The isomorphism}

Let $H$ be a weak Hopf algebra, let $A$ be an algebra, let $p: H \otimes H \rightarrow H \otimes_{H^{R}} H$ be the canonical surjection and let $\rho: H \otimes A \rightarrow A$ and $\sigma: H \otimes_{H^{R}} H \rightarrow A$ be maps. Assume that $\rho$ and $\sigma$ satisfy conditions (1)-(10) (from which $\rho$ and $\varsigma:=\sigma \circ p$ satisfy conditions (2), (4), (11) and (17)-(22)).

Let $i: A \times{ }_{\varsigma}^{\rho} H \rightarrow A \otimes H$ be the canonical inclusion and let $\pi: A \otimes H \rightarrow A \#_{\sigma}^{\rho} H$ be the canonical surjection. Let $\nabla_{\rho}$ be as above of Theorem 1.15. Since, for $a \in A$ and $h \in H$,

$$
\left(\pi \circ \nabla_{\rho}\right)(a \otimes h)=a\left(h^{(1)} \cdot 1_{A}\right) \otimes_{H^{L}} h^{(2)}=a \cdot \Pi^{L}\left(h^{(1)}\right) \otimes_{H^{L}} h^{(2)}=a \otimes_{H^{L}} \Pi^{L}\left(h^{(1)}\right) h^{(2)}=a \otimes_{H^{L}} h,
$$

we have $\pi \circ \nabla_{\rho}=\pi$. Clearly we also have $\nabla_{\rho} \circ i=i$.

Proposition 2.8. The map $\psi: A \times_{\varsigma}^{\rho} H \rightarrow A \#_{\sigma}^{\rho} H$, defined by $\psi:=\pi \circ i$, is a left A-linear and right $H$-colinear algebra isomorphism.

Proof. Let $a, b \in A$ and $h, k \in H$. Since

$$
\begin{aligned}
\psi(a \times h) \psi(b \times k) & =\left(a\left(h^{(1)} \cdot 1_{A}\right) \otimes_{H^{L}} h^{(2)}\right)\left(b\left(k^{(1)} \cdot 1_{A}\right) \otimes_{H^{L}} k^{(2)}\right) \\
& =a\left(h^{(1)} \cdot 1_{A}\right)\left(h^{(2)} \cdot\left(b\left(k^{(1)} \cdot 1_{A}\right)\right)\right) \sigma\left(h^{(3)}, k^{(2)}\right) \otimes_{H^{L}} h^{(4)} k^{(3)} \\
& =a\left(h^{(1)} \cdot\left(b\left(k^{(1)} \cdot 1_{A}\right)\right)\right) \sigma\left(h^{(2)}, k^{(2)}\right) \otimes_{H^{L}} h^{(3)} k^{(3)} \\
& =a\left(h^{(1)} \cdot b\right)\left(h^{(2)} \cdot\left(k^{(1)} \cdot 1_{A}\right)\right) \sigma\left(h^{(3)}, k^{(2)}\right) \otimes_{H^{L}} h^{(4)} k^{(3)} \\
& =a\left(h^{(1)} \cdot b\right) \sigma\left(h^{(2)}, k^{(1)}\right)\left(h^{(3)} k^{(2)} \cdot 1_{A}\right) \otimes_{H^{L}} h^{(4)} k^{(3)}
\end{aligned}
$$




$$
\begin{aligned}
& =\psi\left(a\left(h^{(1)} \cdot b\right) \varsigma\left(h^{(2)} \otimes k^{(1)}\right) \times h^{(3)} k^{(2)}\right) \\
& =\psi((a \times h)(b \times k)) .
\end{aligned}
$$

and

$$
\psi\left(1_{A} \times 1\right)=1^{(1)} \cdot 1_{A} \otimes_{H^{L}} 1^{(2)}=\left(1^{(1)} \cdot 1_{A}\right) \cdot 1^{(2)} \otimes_{H^{L}} 1=\left(1^{(1)} \cdot 1_{A}\right)\left(1^{(2)} \cdot 1_{A}\right) \otimes_{H^{L}} 1=1_{A} \otimes_{H^{L}} 1,
$$

the map $\psi$ is a unitary algebra morphism. It is clear that it is also left $A$-linear and right $H$-colinear. Let $l \in H^{L}, h \in H$ and $a \in A$. Since, by item (3) and [7, Equality (2.7a)],

$\nabla_{\rho}(a \cdot l \otimes h)=a\left(l \cdot 1_{A}\right)\left(h^{(1)} \cdot 1_{A}\right) \otimes h^{(2)}=a\left(l h^{(1)} \cdot 1_{A}\right) \otimes h^{(2)}=a\left(l^{(1)} h^{(1)} \cdot 1_{A}\right) \otimes l^{(2)} h^{(2)}=\nabla_{\rho}(a \otimes l h)$, there exists $\phi: A \#_{\sigma}^{\rho} H \rightarrow A \times_{\varsigma}^{\rho} H$ such that $i \circ \phi \circ \pi=\nabla_{\rho}$. The computations

$$
i \circ \phi \circ \psi=i \circ \phi \circ \pi \circ i=\nabla_{\rho} \circ i=i \quad \text { and } \quad \psi \circ \phi \circ \pi=\pi \circ i \circ \phi \circ \pi=\pi \circ \nabla_{\rho}=\pi \text {, }
$$

prove that $\phi$ and $\psi$ are inverses one of each other.

Remark 2.9. The isomorphism introduced in the previous proposition is natural in an evident sense.

Example 2.10. Let $k$ be a field and $K:=k \times k$. Let $C:=\{1, g\}$ be the cyclic order 2 and let $H:=K \otimes k[C] \otimes K$. For $a, b, c, d \in k$, we set $\lambda_{a b}^{c d}:=(a, b) \otimes 1 \otimes(c, d)$ and $G_{a b}^{c d}:=(a, b) \otimes g \otimes(c, d)$. The $k$-vector space $H$ is a weak Hopf algebra via the multiplication defined by

$$
\lambda_{a b}^{c d} \lambda_{a^{\prime} b^{\prime}}^{c^{\prime} d^{\prime}}:=\lambda_{a a^{\prime}, b b^{\prime}}^{c c^{\prime}, d d^{\prime}}, \quad \lambda_{a b}^{c d} G_{a^{\prime} b^{\prime}}^{c^{\prime} d^{\prime}}:=G_{a a^{\prime}, b b^{\prime}}^{d c^{\prime}, c d^{\prime}}, \quad G_{a b}^{c d} \lambda_{a^{\prime} b^{\prime}}^{c^{\prime} d^{\prime}}:=G_{a b^{\prime}, b a^{\prime}}^{c c^{\prime}, d d^{\prime}} \quad \text { and } \quad G_{a b}^{c d} G_{a^{\prime} b^{\prime}}^{c^{\prime} d^{\prime}}:=\lambda_{a b^{\prime}, b a^{\prime}}^{d c^{\prime}, c d^{\prime}},
$$

and the comultiplication defined by

$$
\Delta\left(\lambda_{a b}^{c d}\right):=\lambda_{a b}^{10} \otimes \lambda_{10}^{c d}+\lambda_{a b}^{01} \otimes \lambda_{01}^{c d} \quad \text { and } \quad \Delta\left(G_{a b}^{c d}\right):=G_{a b}^{10} \otimes G_{01}^{c d}+G_{a b}^{01} \otimes G_{10}^{c d} .
$$

The unit is $\lambda_{11}^{11}$, the counit is the map $\epsilon: H \rightarrow k$ given by $\epsilon\left(\lambda_{a b}^{c d}\right)=a c+b d$ and $\epsilon\left(G_{a b}^{c d}\right)=a d+b c$, and the antipode is the map $S: H \rightarrow H$ given by $S\left(\lambda_{a b}^{c d}\right)=\lambda_{c d}^{a b}$ and $S\left(G_{a b}^{c d}\right)=G_{c d}^{a b}$. A straightforward computation shows that the maps $\Pi^{L}: H \rightarrow H$ and $\Pi^{R}: H \rightarrow H$ are given by

$$
\Pi^{L}\left(\lambda_{a b}^{c d}\right)=\lambda_{a c, b d}^{11}, \quad \Pi^{L}\left(G_{a b}^{c d}\right)=\lambda_{a d, b c}^{11}, \quad \Pi^{R}\left(\lambda_{a b}^{c d}\right)=\lambda_{11}^{a c, b d} \quad \text { and } \quad \Pi^{R}\left(G_{a b}^{c d}\right)=\lambda_{11}^{b c, a d} .
$$

The map $\rho: H \otimes K \rightarrow K$, defined by

$$
\lambda_{a b}^{c d} \cdot(x, y):=(a x c, b y d) \text { and } G_{a b}^{c d} \cdot(x, y):=(a x d, b y c),
$$

where $h \cdot(x, y)$ denotes $\rho(h)(x, y)$ satisfies conditions $(1)-(4)$; and the map $\sigma: H \otimes_{H^{R}} H \rightarrow K$, defined by

$$
\begin{array}{ll}
\sigma\left(\lambda_{a b}^{c d}, \lambda_{a^{\prime} b^{\prime}}^{c^{\prime} d^{\prime}}\right):=\left(a a^{\prime} c c^{\prime}, b b^{\prime} d d^{\prime}\right), & \sigma\left(\lambda_{a b}^{c d}, G_{a^{\prime} b^{\prime}}^{c^{\prime} d^{\prime}}\right):=\left(a a^{\prime} c d^{\prime}, b b^{\prime} d c^{\prime}\right), \\
\sigma\left(G_{a b}^{c d}, \lambda_{a^{\prime} b^{\prime}}^{c^{\prime} d^{\prime}}\right):=\left(a a^{\prime} d d^{\prime}, b b^{\prime} c c^{\prime}\right), & \sigma\left(G_{a b}^{c d}, G_{a^{\prime} b^{\prime}}^{c^{\prime} d^{\prime}}\right):=\left(a a^{\prime} d c^{\prime}, b b^{\prime} c d^{\prime}\right),
\end{array}
$$

satisfies conditions (5)-(9). Moreover $\sigma$ is invertible in the sense of Definition 1.8 . Since $\sigma$ does not fulfill condition (10), this shows that condition (10) in Theorem 2.1 can not be eliminated.

\section{References}

[1] J. N. Alonso Álvarez, J. M. Fernández Vilaboa, J. M. López López, R. González Rodríguez, and A. B. Rodríguez Raposo, Weak Hopf algebras with projection and weak smash bialgebra structures, J. Algebra 269 (2003), no. 2, 701-725.

[2] J. N. Alonso Álvarez, J. M. Fernández Vilaboa, R. González Rodríguez, and A. B. Rodríguez Raposo, Weak C-cleft extensions and weak Galois extensions, Journal of Algebra 299 (2006), no. 1, 276-293.

[3] _ Weak C-cleft extensions, weak entwining structures and weak Hopf algebras, J. Algebra 284 (2005), no. 2, 679-704, DOI 10.1016/j.jalgebra.2004.07.043. MR2114575 
[4] J. N. Alonso Álvarez, J. M. Fernández Vilaboa, R. González Rodríguez, and A. B. Rodríguez Raposo, Crossed products in weak contexts, Appl. Categ. Structures 18 (2010), no. 3, 231-258, DOI 10.1007/s10485-008-9139-2. MR2640214 (2011d:18009)

[5] JN Alonso Álvarez and R González Rodríguez, Crossed products for weak Hopf algebras with coalgebra splitting, Journal of Algebra 281 (2004), no. 2, 731-752.

[6] Gabriella Böhm and Tomasz Brzeziński, Cleft extensions of Hopf algebroids, Applied Categorical Structures 14 (2006), no. 5-6, 431-469.

[7] Gabriella Böhm, Florian Nill, and Kornel Szlachányi, Weak Hopf Algebras, I. Integral Theory and $C^{*}$-Structure, J. Algebra 221 (1999), no. 2, 385-438.

[8] Gabriella Böhm, Florian Nill, and Kornel Szlachányi, Weak Hopf Algebras, II. Representation theory, dimensions and the Markov trace, J. Algebra 233 (2000), 156-212.

[9] J. M. Fernández Vilaboa, R. González Rodríguez, and A. B. Rodríguez Raposo, Preunits and weak crossed products, Journal of Pure and Applied Algebra 213 (2009), 2244-2261.

[10] Jorge A. Guccione, Juan J. Guccione, and Christian Valqui, Cleft extensions of weak Hopf algebras (2019), available at arXiv:1811.02909

[11] Ling Liu, Bing-liang Shen, and Shuan-hong Wang, On weak crossed products of weak Hopf algebras, Algebras and Representation Theory 16 (2013), no. 3, 633-657.

[12] Rodríguez Raposo and Ana Belén, Crossed products for weak Hopf algebras, Comm. Algebra 37 (2009), no. 7, 2274-2289, DOI 10.1080/00927870802620274. MR2536918

Departamento de Matemática, Facultad de Ciencias Exactas y Naturales-UBA, Pabellón 1-Ciudad Universitaria, Intendente Guiraldes 2160 (C1428EGA) Buenos Aires, Argentina.

Instituto de Investigaciones Matemáticas "Luis A. Santaló", Facultad de Ciencias Exactas y Naturales-UBA, Pabellón 1-Ciudad Universitaria, Intendente Guiraldes 2160 (C1428EGA) Buenos Aires, ARgentina.

E-mail address: vander@dm.uba.ar

Departamento de Matemática, Facultad de Ciencias Exactas y Naturales-Uba, Pabellón 1-Ciudad Universitaria, Intendente Guiraldes 2160 (C1428EGA) Buenos Aires, Argentina.

Instituto Argentino de Matemática-COnicet, Savedra 15 3er piso, (C1083ACA) Buenos Aires, Argentina.

E-mail address: jjgucci@dm.uba.ar 